\title{
Pain experiences and intrapersonal change among patients with chronic non-cancer pain after using a pain diary: a mixed-methods study
}

This article was published in the following Dove Medical Press journal: Journal of Pain Research

\author{
Fa-ngam Charoenpol' \\ Nuj Tontisirin ${ }^{2}$ \\ Borwornsom Leerapan ${ }^{3}$ \\ Rattaphol Seangrung ${ }^{2}$ \\ Roderick J Finlayson ${ }^{4}$ \\ 'Department of Anesthesiology, \\ Faculty of Medicine, Khon Kaen \\ University, Khon Kaen, Thailand; \\ 2Department of Anesthesiology, \\ Faculty of Medicine Ramathibodi \\ Hospital, Mahidol University, \\ Bangkok, Thailand; ${ }^{3}$ Department \\ of Community Medicine, Faculty \\ of Medicine Ramathibodi Hospital, \\ Mahidol University, Bangkok, Thailand; \\ ${ }^{4}$ Department of Anesthesia, Alan \\ Edwards Pain Management Unit, \\ McGill University, Montreal, QC, \\ Canada
}

Correspondence: Nuj Tontisirin Department of Anesthesiology, Faculty of Medicine Ramathibodi Hospital, Mahidol University, 270 Rama VI Road, Ratchathewi, Bangkok 10400, Thailand Tel +9866894974667

Email nuj.ton@mahidol.ac.th
Objective: Pain diaries are a valuable self-assessment tool; however, their use in chronic noncancer pain has received limited attention. In this study, we examined the effect of pain diary use on pain intensity, interference, and intrapersonal change in patients with chronic non-cancer pain. Method: A convergent mixed-methods design was used to prospectively evaluate a cohort of 72 patients. Daily pain intensity and weekly pain-interference were self-reported using pain diaries for a 4-week period. Outcomes were assessed by examining changes in pain scores (primary outcome) as well as the Brief Pain Inventory and Short-form McGill Pain Questionnaire-2. In addition, qualitative data obtained from pain diary entries and focus-group interviews were analyzed using thematic content analysis.

Results: Pain intensity and average pain scores were significantly lower after using the diaries. Participants reported less pain interference in mood, walking ability, normal work, and enjoyment of life. No differences were found in SF-MPQ-2 scores. Qualitative analysis indicated that better pain recognition and more effective communication with care providers led to improved self-management and more effectual treatment plans.

Conclusion: Use of a pain diary in patients with chronic non-cancer pain was associated with reduced pain intensity and improved mood as well as function. Further controlled trials examining the long-term effects of pain diaries are warranted.

Keywords: pain diary, chronic non-cancer pain, pain experience, interpersonal change, mixedmethods research

\section{Introduction}

Comprehensive assessment of patients suffering from chronic pain includes evaluation of the sensory, affective, and cognitive domains. Although this process is a critical precursor to the formulation of an effective treatment plan, limited clinical resources and prioritization of the sensory aspect often result in important elements such a pain recognition and coping skills being neglected. Self-reported assessment tools such as pain diaries are easily implemented and have been shown to have a high reliability, validity, and utility. ${ }^{1-7}$ Because they allow patients to monitor fluctuation in their daily pain levels as well as the effect of therapeutic interventions, pain diaries can enhance the sense of self-control and facilitate communication with caregivers. ${ }^{2,3}$ While this has been demonstrated in individuals with chronic cancer-related pain, findings have been less positive in those with acute neck and back pain. ${ }^{8,9}$ In contrast, little is known about pain diary use in patients with chronic non-cancer pain. We therefore undertook a study to evaluate the effect of this tool in subjects suffering from chronic non-cancer 
pain and theorized that pain diary use could be associated with improved pain management in this population.

\section{Material and methods Study design}

The present study used a mixed-methods design with using a concurrent triangulation approach ${ }^{10-13}$ to explore pain experiences and investigate intrapersonal change before and after the use of a pain diary and we simultaneously collected both quantitative and qualitative data.

Statistical analyses were conducted to determine differences in participants' pain intensity and interference before and 4 weeks after using a pain diary. Thematic content analysis was used to synthesize the emerging themes from the pain diaries and findings from focus-group interviews. Then, the quantitative and qualitative were analyzed to determine if there was convergence, differences, or some combinations.

\section{Participants}

This study was approved by the Committee on Human Rights Related to Research Involving Human Subjects of the Faculty of Medicine Ramathibodi Hospital, Mahidol University (EC ID: ID 08-59-04) and was registered to Thai Clinical Trial Registry (TCTR 20160906001). This study was conducted in accordance with the Declaration of Helsinki. In total, 72 patients with chronic non-cancer pain who received followup and treatment at the Ramathibodi Hospital's Pain Clinic in Bangkok, Thailand between June 1, 2016 and December 31, 2016 were enrolled after giving written and informed consent. Included patients were those aged 18-65 years presenting noncancer pain for more than 3 months, fluent in the Thai language. Patients who were unable to complete the 4-week follow-up, or who requested withdrawal from the study, were excluded.

\section{Sample size calculation}

As few studies involving use of a pain diary in chronic non-cancer pain from different causes and locations were available, the sample size calculation was based on a previous study examining cancer-related pain ${ }^{3}$ that found average pain intensity scores before and after using pain diary use of 2.76 (SD 1.64) and 2.15 (SD 1.67), respectively. Using an effect size of $36 \%$, alpha error of 0.05 and beta error of 0.20 , a sample size of 56 patients was calculated. Allowing for a potential drop out of $\sim 30 \%$, the total sample size required was 72 participants.

\section{Quantitative data collection}

Demographic data were recorded including age, gender, pain history (diagnosis, location), pain category (nociceptive pain, neuropathic pain, or mixed), duration of pain, education level, employment, and medication.

Other quantitative data were collected from three sources:

1. Thai Brief Pain Inventory (Thai-BPI)

2. Thai Short-Form McGill Pain Questionnaire-2 (Thai-SF-MPQ-2)

3. Daily and weekly quantitative data, written in pain diary as shown below.

Participants completed the Thai-BPI and the Thai-SF-MPQ-2 at their first visit before receiving a pain diary (baseline), and at 4 weeks follow-up.

\section{Thai BPI ${ }^{14}$}

The Thai BPI includes the following elements:

1. body pain mapping

2. pain intensity: 1) worst pain, 2) least pain, 3) average pain intensity during last 24 hours, and 4 ) pain right now (rated on a scale from 0 to $10 ; 0=$ no pain, $10=$ worst possible pain)

3. pain interference in: 1) normal work, 2) walking ability, 3) general activity, 4) enjoyment of life, 5) mood, 6) relationship, and 7$)$ sleep $(0=$ no interference, $10=$ most disturbance)

4. a question about current pain medication (name, indication, dose, route, and start date)

5. a question about how the participant rated their improvement in the past 24 hours.

\section{Thai SF-MPQ-2 ${ }^{15}$}

The Thai SF-MPQ-2 includes 22 pain characteristics that represent four aspects of pain, each rated on a numerical scale from 1 to 10 :

1. continuous pain (six characteristics: throbbing, cramping, gnawing, aching, heavy pain, and tender)

2. intermittent pain (six characteristics: shooting, stabbing, sharp, splitting, electrical-shock, and piercing)

3. neuropathic pain (six characteristics: hot-burning, coldfreezing, pain caused by light touch, itching, tingling, or pins and needles, numbness)

4. affective aspect (four characteristics: tiring-exhausting, sickening, fearful, and punishing-cruel).

Both tools complement each other as they examine different pain aspects of the pain experience. For example, BPI measures pain interference and average pain score, whereas the SF-MPQ-2 examines the nociceptive component of pain (both continuous and intermittent), as well as the neuropathic and affective components. 


\section{Daily and weekly quantitative data}

Daily data included average pain intensity and frequency of breakthrough medication requirement. Weekly data were disturbance from pain in activity, mood, walking ability, daily activity, relationship, sleep, and happiness (0-10 scale, $0=$ no interference, $10=$ most disturbing).

\section{Quantitative data analysis}

Demographic data (including age, gender, pain history, and Thai BPI and Thai SF-MPQ-2 scores) were recorded and analyzed using PASW Statistics (SPSS) version 18.0. General information and pretest-posttest scores are presented as mean, frequency, and percentages as appropriate. Data were analyzed using paired $t$-tests and repeated measures ANOVA. ${ }^{16}$ For multiple comparison, we used repeated measures ANOVA first. If significant, then paired $t$-test with adjustments for multiple comparisons was performed.

\section{Qualitative data collection}

Participants provided qualitative data regarding their "pain experiences" by responding to five open-ended questions in their pain diary each week. The open-ended questions investigated: 1) how participants perceived their pain, 2) how they managed their pain, 3) their sense of control of their pain, 4) how they communicated with others about their pain, and 5) provided space for any other comments about their pain during that week.

Study participants who were able to provide the best written feedback regarding their experience with the pain diary were selected to be key informants in two focus group interviews. Selected key informants were divided into two groups, a first one consisting of individuals who had entered a large amount of information in their pain diaries and noted that they had benefits in pain management after using the pain diary. A second group consisted of patients who had only completed quantitative items, provided little-to-no qualitative information, gave similar ratings for most quantitative items, or mentioned that they did not get any benefits from the pain diary.

Three investigators reviewed participants' pain diary entries before creating semistructured questions for further discussion with focus group participants. The list of questions used in the focus group interviews is shown in Supplementary S1. The interviews for each group took about 45 minutes and were voice-recorded and transcribed verbatim. In addition to the verbatim transcripts, each investigator observed and made notes about how participants talked and behaved during the interviews, including nonverbal body language. All three investigators subsequently compared their notes to triangulate their observations and interpretations.

\section{Qualitative data analysis}

Qualitative data extracted from the pain diaries, verbatim transcripts, and investigators' memos were used for thematic content analysis. All text data were entered and processed with the qualitative data analysis software ATLAS.ti version 7.0. ${ }^{17}$ Using the protocol described by Pope et al, the thematic content analysis process included: 1) familiarization, or immersion in the raw data to list key ideas, 2) identifying a thematic framework (key concepts and themes), 3) indexing, or applying the thematic framework/index systematically to all data in textual form by annotating the transcripts with numerical codes, 4) rearranging the data according to the appropriate part of the thematic framework to which they related and forming charts, and 5) mapping and interpretation using the charts to define concepts and map the range and nature of phenomena. ${ }^{18}$ The findings were translated to English by the first three authors.

\section{Results Demographic data}

In total, 67 participants (93.1\%) completed the pain diary for 4 weeks and returned it to the clinic; however, only 61 diaries $(91.0 \%)$ were completed with daily and weekly data (Figure 1). The majority of participants were female $(\mathrm{n}=40$, $59.7 \%$ ) with an average age of $47 \pm 12$ years. Neuropathic pain was the most common pain category $(n=32,47.8 \%)$, followed by mixed pain $(n=21,31.3 \%)$, and nociceptive pain $(n=14$, 20.9\%). Most participants had pain in more than three sites $(\mathrm{n}=13 ; 19 \%)$ with a duration of 3 months to 1 year $(\mathrm{n}=26$; $39 \%)$, had a high school ( $\mathrm{n}=27 ; 41 \%)$ or Bachelor's degree $(\mathrm{n}=31 ; 46 \%)$. The most commonly used pain regimen was a combination of a weak opioids and anticonvulsant $(\mathrm{n}=16$; 23\%) (Table 1).

\section{Quantitative results}

There was no statistical difference in Thai SF-MPQ-2, either total or subscale scores, before and after using the pain diary. However, the mean score for the affective subscale was higher than the other subscales (continuous, intermittent, and neuropathic pain) (Table 2). Thai BPI score showed that participants reported lower current and average pain scores, and significantly less pain interference in mood, walking ability, normal work, and enjoyment of life after pain diary use (Table 3). Analysis of daily data from pain diaries $(n=61)$ showed there was no difference in pain intensity before and after using the pain diary. However, there was a trend toward 
72 participants received pain diary and filled out Thai BPI, Thai SF-MPQ-2

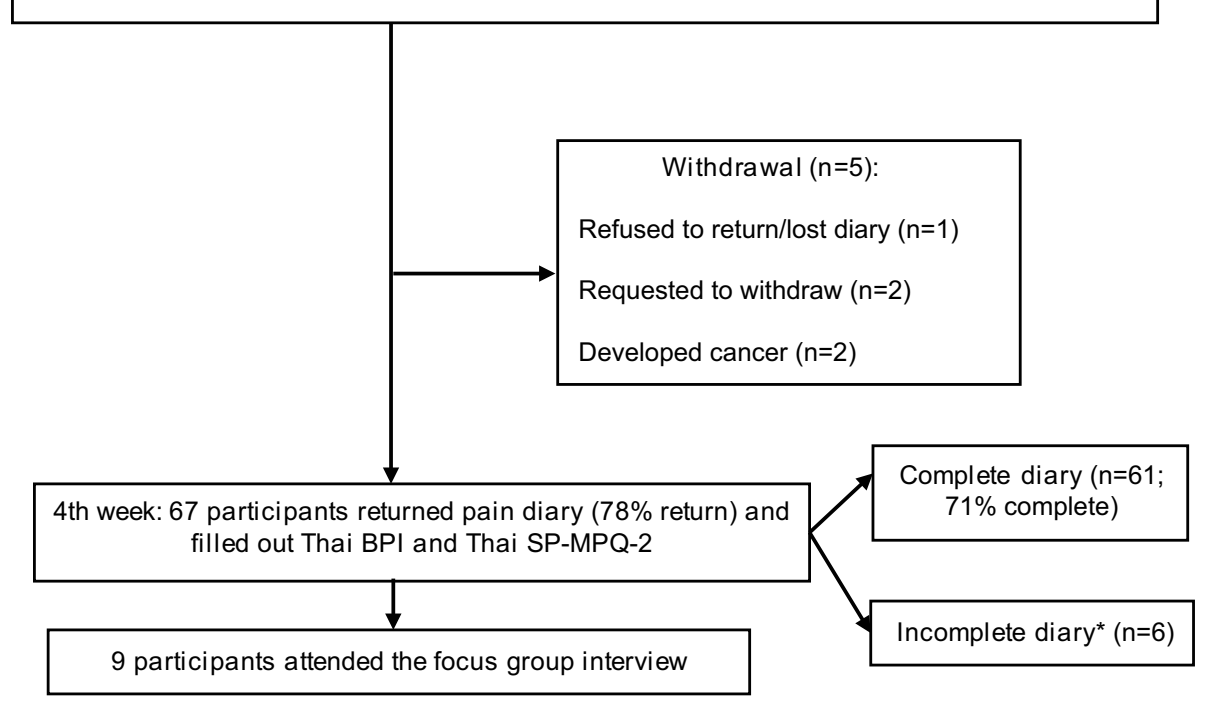

Figure I Patient selection flowchart.

Notes: Complete diary: completely filled out both quantitative, daily and weekly data, as well as qualitative data. *Incomplete diary: filled out qualitative data but incompletely filled out daily and weekly quantitative data.

Abbreviations: Thai-BPI, Thai Brief Pain Inventory; Thai SF-MPQ-2, Thai Short-Form McGill Pain Questionnaires-2.

reduced pain intensity after using the pain diary $(P=0.578)$ (Figure 2). Weekly data from pain diaries ( $\mathrm{n}=61)$ showed no statistically significant difference in pain interference (Table 4).

\section{Qualitative results}

Four pain experience themes emerged among patients with chronic non-cancer pain after using the pain diary. First, the pain diary facilitated participants' self-management through better recognition and understanding of their pain. Second, the pain diary facilitated participants' self-management through better patient-provider communication and support obtained from providers. Third, the pain diary provided a channel to relieve mood disturbance and vent negative thoughts. Finally, although the pain diary was helpful for many participants, some reported it was not helpful.

\section{Facilitating participants' self-management through better recognition and understanding of their pain}

Participants perceived their pain as having different intensities and characteristics each day. Some participants had not recognized and remembered these aspects before using the diary and it helped them recognize these pain patterns. Participants were also better able to describe their pain characteristics by choosing from the 22 pain characteristics listed in the Thai-SF-MPQ-2 and could therefore report their pain to their physician more accurately. In particular, those with multiple pain characteristics and varied pain patterns reported that the pain diary helped them remember their pain patterns.

I can show the doctor how the pain changes, when previously, I usually could remember for just 2-3 weeks before an appointment. [Participant 1, focus group 1].

At that time, we were not able to distinguish the pain properly, but the pain diary had an option to choose. [Participant 3 , focus group 1].

Other participants did not experience such benefits.

When I do not have pain and opened it [the pain diary], I found that I had a lot of pain on that day. [Participant 6, focus group 2].

That week, I knew that my left arm pain was relieved, but the pain on my neck and back were still there. [Participant 7, focus group 2].

Participants used the questionnaires in the diary, which seem to be guiding some patients to consider their pain characteristics and patterns (researcher's observational note).

Additionally, using the pain diary led to participants' to better understanding their pain experiences, particularly what aggravated or alleviated their pain. As a result, they could modify their behaviors or manage their pain themselves (eg, taking regular physical exercise or avoiding activities that might elicit pain). These self-management skills led to better pain control and less pain interference. 
I noted in the diary that pain aggravated by activities limited my functions [Participant 1, focus group 1].

The drug just relieved the pain, and not cured it. It made me felt depressed. I tried to do activities that could relieve

Table I Demographic data $(n=67)$

\begin{tabular}{|c|c|c|}
\hline \multicolumn{2}{|l|}{ Characteristic } & \multirow{2}{*}{$\begin{array}{l}\text { Number (\%) } \\
27(40)\end{array}$} \\
\hline Gender & Male & \\
\hline & Female & $40(60)$ \\
\hline Age & Mean \pm SD (years) & $46 \pm 12$ \\
\hline \multirow[t]{3}{*}{ Type of pain } & Nociceptive & $14(21)$ \\
\hline & Neuropathic & $32(48)$ \\
\hline & Mixed & $2 I(3 I)$ \\
\hline \multirow[t]{11}{*}{ Location } & 3 or more sites & $13(19)$ \\
\hline & Buttock and leg & II (16) \\
\hline & 2 sites & $10(15)$ \\
\hline & Back & $7(I I)$ \\
\hline & Shoulder/arm & $5(7.5)$ \\
\hline & Abdomen & $5(7.5)$ \\
\hline & Leg & $5(7.5)$ \\
\hline & Neck and arm & $4(6)$ \\
\hline & Head (face) & $4(6)$ \\
\hline & Foot & $2(3)$ \\
\hline & Pelvis & $\mathrm{I}(\mathrm{I} .5)$ \\
\hline \multirow[t]{4}{*}{ Duration of pain } & $>3$ months-I year & $26(39)$ \\
\hline & $1-5$ years & $23(34)$ \\
\hline & $6-10$ years & $11(16)$ \\
\hline & $>10$ years & 7 (II) \\
\hline \multirow[t]{5}{*}{ Education level } & None & I (2) \\
\hline & Primary school & $18(26)$ \\
\hline & Secondary school & $12(17)$ \\
\hline & Diploma or bachelor degree & $27(4 I)$ \\
\hline & Master degree or $\mathrm{PhD}$ & $9(14)$ \\
\hline \multirow[t]{3}{*}{ Employment } & Working & $31(46)$ \\
\hline & Unemployed & $18(27)$ \\
\hline & Retired & $18(27)$ \\
\hline \multirow[t]{8}{*}{ Medications used } & NSAIDs & $I(I)$ \\
\hline & Opioids & $6(9)$ \\
\hline & Anticonvulsant & $12(18)$ \\
\hline & Antidepressant & $5(8)$ \\
\hline & Weak opioids + anticonvulsant & $16(23)$ \\
\hline & Weak opioids + antidepressant & $5(8)$ \\
\hline & $\begin{array}{l}\text { Weak opioids }+ \text { antidepressant } \\
+ \text { anticonvulsant }\end{array}$ & $12(18)$ \\
\hline & $\begin{array}{l}\text { Antidepressant + } \\
\text { anticonvulsant }\end{array}$ & $10(15)$ \\
\hline
\end{tabular}

the pain such as exercise, praying, or studying the Buddha's teaching. [Participant 5, focus group 1].

Now I understand more about my pain and how to manage it. [Participant 8 , focus group 2].

\section{Facilitating participants' self-management through} better patient-provider communication and support from providers

Participants reported the pain diary was helpful in terms of communicating pain intensity, pain characteristics, patterns, and adverse effects from pain medication with their physicians, as well as showing how the pain had changed from their last visit. Some participants also explained their pain location to their physician using the body mapping in the pain diary. Participants' improved recognition and understanding of their pain helped them communicate issues more clearly to their physicians, which in turn allowed physicians to provide more effective guidance, education, planning, and pain management. At the same time, participants felt more understood and appeared to have better self-esteem, which further encouraged them to manage their pain themselves.

Now I can explain where the pain is using body mapping.

[Participant 1, focus group 1].

This was like talking about symptoms every day, instead of once a month when I visited the doctor. Sometimes, I can't explain my pain to the doctors. But when they read the diary, they will know that I have a different pain each day. [Participant 5, focus group 1].

Pain diary guided me what to talk about with my doctor.

[Participant 6, focus group 2].

Although participants reported the pain diary helped them communicate better with physicians, they perceived their communication with family and friends remained challenging. This may be particularly relevant in Thai or other Asian cultures, where self-expression of emotional stress is less common and less acceptable, even among family members. Participants reported that talking about their pain was annoying to their family and friends and was also futile

Table 2 The comparison of Thai Short-Form McGill Pain Questionnaire-2 $(n=67)$

\begin{tabular}{|l|l|l|l|}
\hline Subscale of pain & Before using diary (mean \pm SD) & After using diary (mean \pm SD) & $P$-value \\
\hline Overall & $2.82 \pm 0.23$ & $2.77 \pm 0.24$ & 0.719 \\
\hline I. Affective & $4.37 \pm 0.35$ & $4.32 \pm 0.35$ & 0.838 \\
\hline 2. Continuous & $2.93 \pm 0.28$ & $2.86 \pm 0.27$ & $0.68 I$ \\
\hline 3. Intermittent & $2.12 \pm 0.24$ & $2.06 \pm 0.29$ & 0.704 \\
\hline 4. Neuropathic & $1.86 \pm 0.21$ & $1.84 \pm 0.23$ & 0.878 \\
\hline
\end{tabular}


Table 3 The comparison of Thai Brief Pain Inventory $(n=67)$

\begin{tabular}{|l|l|l|l|l|}
\hline Thai Brief Pain Inventory item & Pretest score (mean \pm SD) & Posttest score (mean \pm SD) & P-value & Effect size (\%) \\
\hline Maximum pain score in 24 hours & $5.8 \pm 2.7$ & $5.6 \pm 3.0$ & 0.56 & 5.3 \\
\hline Minimum pain score in 24 hours & $3.2 \pm 2.4$ & $3.3 \pm 2.5$ & 0.72 & -4.1 \\
\hline Average pain score & $4.9 \pm 2.4$ & $4.5 \pm 2.3$ & $0.03^{*}$ & 17.7 \\
\hline Pain score now & $4.7 \pm 2.6$ & $4.2 \pm 2.7$ & $0.04^{*}$ & 18.5 \\
\hline Treatment relief pain & 4.92 .5 & $4.8 \pm 2.8$ & 0.83 & 3.4 \\
\hline General activity & $5.0 \pm 3.0$ & $4.7 \pm 3.0$ & 0.41 & 7.0 \\
\hline Mood & $4.8 \pm 2.8$ & $4.2 \pm 2.8$ & $0.0 I^{*}$ & 20.6 \\
\hline Walking ability & $4.6 \pm 3.1$ & $0.0 \pm 3.0$ & 20.8 \\
\hline Normal work & $5.5 \pm 3.0$ & $4.6 \pm 2.9$ & $0.004^{*}$ & 31.8 \\
\hline Relationship & $3.9 \pm 2.9$ & $3.6 \pm 3.0$ & 0.20 & 8.5 \\
\hline Sleep & $4.8 \pm 3.2$ & $4.4 \pm 3.0$ & 0.22 & 13.5 \\
\hline Enjoyment of life & $5.0 \pm 2.9$ & $4.4 \pm 3.0$ & $0.02^{*}$ & 18.7 \\
\hline
\end{tabular}

Note: $* P$-value $<0.05$.

Pain average

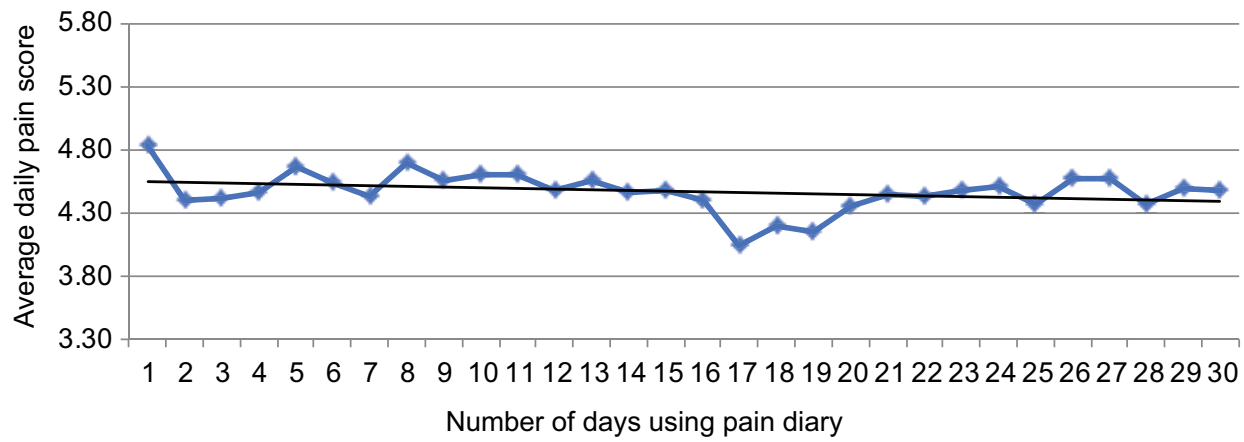

Figure 2 Daily pain score (numerical rating scale: range 0-10).

Table 4 The comparison of weekly data $(n=6 I)$

\begin{tabular}{|l|l|l|l|l|l|l|l|}
\hline $\begin{array}{l}\text { Item (BPI- } \\
\text { questionnaire) }\end{array}$ & $\begin{array}{l}\text { Pretest score } \\
\text { (mean } \pm \text { SD) }\end{array}$ & Ist week & 2nd week & 3rd week & 4th week & $\begin{array}{l}\text { Posttest score } \\
\text { (mean } \pm \text { SD) }\end{array}$ & $\begin{array}{l}\text { Repeated } \\
\text { ANOVA } \\
\text { P-value }\end{array}$ \\
\hline General activity & $4.9 \pm 0.4$ & $4.4 \pm 0.4$ & $4.2 \pm 0.4$ & $4.1 \pm 0.4$ & $4.3 \pm 0.4$ & $4.7 \pm 0.4$ & $0.04 I^{*}$ \\
\hline Mood & $4.7 \pm 0.4$ & $4.3 \pm 0.4$ & $4.0 \pm 0.4$ & $4.0 \pm 0.4$ & $4.5 \pm 0.4$ & $4.2 \pm 0.4$ & 0.094 \\
\hline Walking ability & $4.6 \pm 0.4$ & $3.7 \pm 0.4$ & $3.5 \pm 0.4$ & $3.4 \pm 0.4$ & $3.7 \pm 0.4$ & $3.9 \pm 0.4$ & $0.003^{*}$ \\
\hline Normal work & $5.4 \pm 0.4$ & $4.2 \pm 0.4$ & $4.2 \pm 0.4$ & $4.2 \pm 0.4$ & $4.3 \pm 0.4$ & $4.5 \pm 0.4$ & $0.002^{*}$ \\
\hline Relationship & $3.7 \pm 0.4$ & $3.3 \pm 0.4$ & $3.3 \pm 0.4$ & $3.0 \pm 0.4$ & $3.5 \pm 0.4$ & $3.6 \pm 0.4$ & 0.132 \\
\hline Sleep & $4.9 \pm 0.4$ & $3.8 \pm 0.4$ & $3.7 \pm 0.4$ & $3.7 \pm 0.4$ & $4.1 \pm 0.4$ & $4.4 \pm 0.4$ & $0.002^{*}$ \\
\hline Enjoyment of life & $4.9 \pm 0.4$ & $4.3 \pm 0.4$ & $3.9 \pm 0.4$ & $4.3 \pm 0.4$ & $4.6 \pm 0.4$ & $4.3 \pm 0.4$ & $0.017^{*}$ \\
\hline
\end{tabular}

Notes: $* P$-value $<0.05$ for repeated ANOVA. For the paired $t$-test using adjusted $P$-value $<0.003$, there was no statistical significance.

because people who had not experienced chronic pain could not understand their chronic pain.

Pain is not an interesting topic to frequently talk about with family or colleagues. [Participant 6 , focus group 2].

They might get bored and wonder, while I can walk, work or never take a sick-leave, why my pain is persistent or never goes away. [Participant 7, focus group 2].
I never told them how my pain was severe and how I was suffering. When they saw me smile, they thought I was fine. But people at the pain clinic understand my pain well, and the diary was a tool to show them my pain more easily. [Participant 8, focus group 2].

I had no one to talk to before having been treated at the pain clinic with a diagnosis of fibromyalgia. My husband 
didn't understand me. He said I was normal, just demanding

or seeking attention. [Participant 9, focus group 2].

They were afraid of making their family or other people feel unpleasant (researcher's observational note).

\section{A channel to relieve mood disturbance and vent negative thoughts}

Most participants avoided expressing their emotional stress and accepted their condition. Although they suffered from pain, sorrow, or anger, most participants suppressed their self-expression of these emotions. However, some were able to use the pain diary as a coping mechanism to relieve their mood disturbance and vent negative thoughts. Previously, some participants had attempted to release stress by writing about their feelings, hopes, fears, or self-blame on a piece of paper; later throwing it away in an attempt to forget their pain. After using the pain diary, some participants found it had a similar effect, and began using the diary to write about their pain. This venting effect from writing in the pain diary appeared to lead to less mood disturbance.

When I got the diary, I complain to the diary. [Participant

2 , focus group 1].

When I have vented away from the pain, it's like we've forgotten about this pain. [Participant 8 , focus group 2].

\section{No intrapersonal change after using pain diary in some participants}

After participants had used the pain diary for 4 weeks, we found that most regularly and actively used their pain diary. In total, 56 of 67 participants (84\%) reported they received benefits from using the pain diary, especially in monitoring their pain, which helped them better manage their pain. However, eleven participants (16\%) felt that they did not get any benefits from using the pain diary, and refused to complete it (by not providing answers to qualitative questions or providing little-to-no information).

We found that participants who did not complete the pain diary were likely to be those who had lived with their pain for a long time. This group of patients expressed concern about not changing anything they had already done. For example, they attempted to not talk about their pain, not focus on their pain, and avoid activities that may provoke their pain.

People who have lived with pain for 20 years already know exactly how to protect themselves. [Participant 6 , focus group 2].

Do not need to take notes. It would not be different from day to day. [Participant 7, focus group 2].
When I take notes, I feel that the pain is still there.

[Participant 8, focus group 2].

Why do I have to remember it? It should be better if

I don't focus on the pain. [Participant 6, focus group 2].

Some patients felt that using pain diary was linked with encountering their pain. Some patients used maladaptive coping mechanisms to protect themselves from pain (researcher's observational note).

\section{Integrating quantitative and qualitative data}

Overall, our quantitative and qualitative findings regarding the pain experiences of patients with chronic non-cancer pain after using a pain diary for 4 weeks were consistent. The quantitative data demonstrated that the average and current pain intensity significantly decreased after using the diary for 4 weeks. Similarly, the qualitative data indicated that patients appreciated the process of writing in the pain diary as it helped them to cope better with their pain through various mechanisms. More specifically, our quantitative findings indicated using the pain diary reduced pain interference in mood, walking ability, normal work, and enjoyment of life, and the qualitative findings revealed the pain diary provided a channel to manage their mood disturbance and vent negative thoughts. This particular intrapersonal change in the affective domain is crucial, as the Thai BPI data showed that patients with chronic non-cancer pain had higher affective pain intensity compared with nociceptive or neuropathic pain intensity.

Our qualitative findings revealed details and heterogeneity of participants' pain experiences and intrapersonal changes that could not be captured by statistical analyses. While the quantitative data highlighted overall positive outcomes, the qualitative data suggested pain experiences differ among subgroups of patients with chronic non-cancer pain. Our in-depth focus group interview with patients who actively used the pain diary over the 4-week study period revealed that they had a positive experience and benefited from the process through better recognition and understanding their pain, better communication with care providers, and reduced mood disturbance/negative thoughts through venting. In contrast, the focus group interview with patients who did not actively use the pain diary revealed negative experiences and a perception that they did not receive any benefits from using the pain diary. This heterogeneity of pain experiences between the two subgroups was consistent with the relatively high SD of pain severity and pain-interference as measured by the Thai BPI. 


\section{Discussion}

The present study showed convergence of qualitative and quantitative data in that the majority of participants experienced benefits after using the pain diary in terms of reduced pain intensity and pain interference, as well as improved self-management. However, some patients with chronic non-cancer pain did not benefit from pain diary use. Previous studies showed that beneficial intrapersonal changes after using pain diary can include improved self-management associated with increased recognition or awareness of pain and better communication with caregivers. This appears to be consistent for patients with and without cancer. ${ }^{3,4}$ Schumacher et al demonstrated the usefulness of the PRO-SELF ${ }^{\circ}$ Pain Control Program in patients with cancer, although that study did not specifically investigate intrapersonal changes among patients who did not benefit from pain diary use. ${ }^{4}$

LeMay et al found differences in pain perception and mood interference in patients with cancer when compared to those without. ${ }^{19}$ Patients with chronic non-cancer pain reported more depression and higher pain severity. This was consistent with our finding that patients with chronic non-cancer pain had higher scores on the affective subscale of pain when compared with other subscales. The present study also showed that patients had less mood interference after using the pain diary as a stress-venting tool. Therefore, a pain diary may be useful in reducing mood disturbance, which may be more pronounced in patients with chronic non-cancer pain, especially in Thai or other Asian cultures with limited self-expression.

There was an inconsistency in the comparison of pain intensity between the Thai BPI and Thai SF-MPQ-2. The Thai SF-MPQ-2 discriminates between the psychological and physical components of pain, whereas the Thai BPI represents overall pain intensity. The average pain intensity score on the Thai BPI was close to that for the Thai-SF-MPQ-2 affective subscale. We theorize that participants' reported pain score mainly represented the affective component of pain. However, we did not use a standardized questionnaire to measure mood disorders, such as depression and anxiety. We therefore cannot ascertain how many participants had mood disorders that may have affected their pain intensity.

Our study is one of the first empirical studies to report intrapersonal changes among patients with chronic noncancer pain after using a pain diary and possible explanatory mechanisms for these observations are presented in Figure 3.

Patients with adaptive coping strategies are likely to benefit from a pain diary as a tool to enhance their coping skills. In our study, the pain diary helped such patients to better understand how their behaviors, feelings, and thoughts influenced their pain and how their own efforts to manage their pain in turn influenced their pain experiences. Consequently, this encouraged their self-management. For example, this group of patients more often engaged in exercise or activities that alleviated their pain/stress from pain, as well as learning about goal setting, activity pacing, and altering overly negative thoughts. This is consistent with a recent meta-analysis that suggested training in pain coping skills can play an important role in cognitive behavioral therapy (CBT) for pain management. ${ }^{20}$ Dixon et al discussed three main components of CBT: 1) educational rationale (better understanding pain, thoughts, and behaviors and influencing their efforts on pain), 2) therapist-guided training in cognitive and behavioral coping (relaxation training, realistic goal setting, learning how to overcome negative thoughts), and 3) extensive practice with coping skills and applying those skills to real situations. ${ }^{21}$ A pain diary may be a useful self-guided tool that creates intrapersonal change for pain management similar to those achieved by CBT. For example, in step 1education-rationale; patients understand about their pain (by using pain diary), step 2 - therapist-guided therapy (patients used pain diary to communicate with physicians to guide their treatment plan), and step 3 - extensive practice and apply to real situation (patients used pain diary to monitor their pain in the daily life to adapt the management up to the change of daily activities).

Conversely, patients with maladaptive coping (eg, avoidance) may experience a vicious cycle of worsening pain (Figure 3); these participants typically refused to complete the pain diary, leading to failure of pain management. ${ }^{22}$ This knowledge may help physicians who encounter patients who refuse to use a pain diary (or only provide limited information) to understand more about these patients' hidden pain coping skills. Therefore, CBT and patient-education that encourages patients to use the pain diary might be beneficial in pain management for these patients. Although direct effects vary among patients, a pain diary can be used as a simple tool to help physicians gain a better understanding of their patients' coping strategies, which may lead to more effective pain management plans.

This study had some limitations. First, although the present findings suggest a pain diary may be effective for patients with chronic non-cancer pain, no standardized questionnaire was used to assess pain coping skills, and the categorization and assessment of pain coping skills were inconclusive. Practically, clinical assessment and subjective judgment remain the primary techniques. Future studies should consider using a pain coping screening questionnaire such as the Coping 


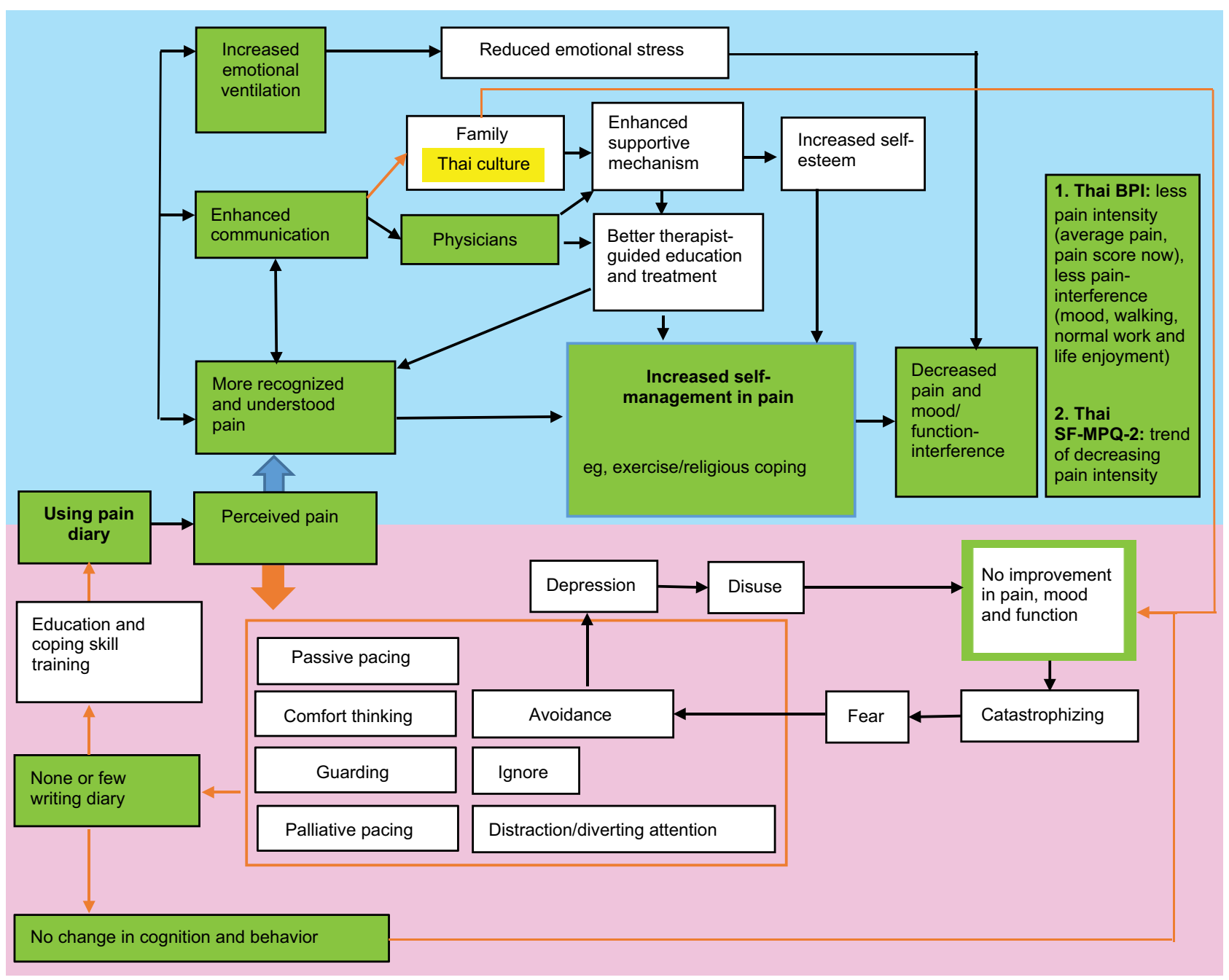

Figure 3 Themes of changes after using pain diary.

Notes: This figure showed hypothesis from triangulating analysis (green boxes) correlated to existing theories (white boxes). After using a pain diary, all patients perceived and subsequently coped with their pain. The pain diary enhanced patients hidden coping skills, including: I) increasing recognition and understanding of their pain, which encouraged better self-management, 2) enhancing communication with physician leading to better therapist-guided education and treatment, and 3) venting of emotional stress, which may reduce mood-interference. Some patients, who did not use the pain diary, had mostly maladaptive coping skills, such as avoidance, which might lead to worsening pain. The coping skill training might help these patients to have more adaptive coping, that may enhance the utility and benefit from a pain diary.

Abbreviations: Thai-BPI, Thai Brief Pain Inventory; Thai-SF-MPQ-2, Thai Short-Form McGill Pain Questionnaire-2.

Strategies Questionnaire or the revised Coping Strategies. ${ }^{23,24}$ In practice, patients with maladaptive coping strategies may be more easily detected by pain coping screening, which would allow physicians to prepare more appropriate pain management plans. Second, the quantitative questionnaires used in this study may not be accurate in detecting the difference of pain interference in every patient. For example, patients with spinal cord injury and paraplegia had difficulty completing the pain interference items relating walking ability. A revised version of the BPI questionnaire that addresses such issues may lead to more valid self-report in future studies. Third, the group of enrolled participants (9\%) that did not return a pain diary might have included patients who did not have pain relief after using the pain diary; this might have influenced the average scores for change in pain intensity and pain interference. Fourth, this study followed patients for only 4 weeks and future studies with longer follow-up periods are needed. Lastly, most the participants were longterm patients of our pain clinic and exposure to prior pain education, which was not assessed in the current protocol, may have had an effect on the study findings.

\section{Conclusion}

Beneficial intrapersonal changes were observed after pain diary use, including enhanced self-management skills which were associated with improved self-recognition, 
communication skills, as well as reduced mood disturbances and negative thoughts. These benefits led to less pain intensity and pain interference. A subset of patients with maladaptive coping skills showed no benefit from pain diary use. Further controlled trials examining the long-term effects of pain diaries are warranted.

\section{Data sharing statement}

Individual deidentified participants data that underlie the results reported in this article will be shared (text, tables, figures, and appendices). The study protocol will also be available. All data will be available immediately and ending 5 years following publication; for researchers who provide a methodologically sound proposal, in particular, for individual participants data meta-analysis. The proposals should be directed to the corresponding author at doctornuj@gmail. com. To gain access, data requestors will need to sign a data access agreement.

\section{Acknowledgments}

The authors are very grateful to all the patients who actively participated in the program and thank the staff of Ramathibodi Hospital's Pain Clinic, particularly Rojnarin Komonhirun, Med, and Wareeya Vongspanich, MS, who provided assistance and coordination support. The authors also thank Audrey Holmes, MA, from Edanz Group (www.edanzediting. $\underline{\mathrm{com} / \mathrm{ac}}$ ) for editing a draft of this manuscript.

\section{Disclosure}

The authors report no conflicts of interest in this work.

\section{References}

1. Linton SJ, Shaw WS. Impact of psychological factors in the experience of pain. Phys Ther. 2011;91(5):700-711.

2. Richardson JC, Ong BN, Sim J. Experiencing and controlling time in everyday life with chronic widespread pain: a qualitative study. $B M C$ Musculoskelet Disord. 2008;9(1):3.

3. de Wit R, van Dam F, Hanneman M, et al. Evaluation of the use of a pain diary in chronic cancer pain patients at home. Pain. 1999;79(1):89-99.

4. Schumacher KL, Koresawa S, West C, et al. The usefulness of a daily pain management diary for outpatients with cancer-related pain. Oncol Nurs Forum. 2002;29(9):1304-1313.
5. Stewart WF, Lipton RB, Kolodner KB, Sawyer J, Lee C, Liberman JN. Validity of the Migraine Disability Assessment (MIDAS) score in comparison to a diary-based measure in a population sample of migraine sufferers. Pain. 2000;88(1):41-52.

6. Stinson JN, Stevens BJ, Feldman BM, et al. Construct validity of a multidimensional electronic pain diary for adolescents with arthritis. Pain. 2008;136(3):281-292.

7. Dampier C, Ely B, Brodecki D, O'Neal P. Characteristics of pain managed at home in children and adolescents with sickle cell disease by using diary self-reports. J Pain. 2002;3(6):461-470.

8. Ferrari R, Louw D. Effect of a pain diary use on recovery from acute whiplash injury: a cohort study. J Zhejiang Univ Sci B. 2013;14(11):1049-1053.

9. Ferrari R. Effect of a pain diary use on recovery from acute low back (lumbar) sprain. Rheumatol Int. 2015;35(1):55-59.

10. Morgan DL. Practical strategies for combining qualitative and quantitative methods: applications to health research. Qual Health Res. 1998;8(3):362-376.

11. Tashakkori A, Teddlie C. Mixed Methodology: Combining Qualitative and Quantitative Approaches. Vol. 46. Thousand Oaks, CA: Sage; 1998:185.

12. Creswell JW, Plano Clark VL, Guttman M, Hanson W. Advanced mixed methods research designs. In: Tashakkori A, Teddlie C. editors. Handbook on Mixed Methods in Social and Behavioral Research. Thousand Oaks, CA: Sage; 2003:209-240.

13. Creswell JW. Research Design: Qualitative, Quantitative, and Mixed Methods Approaches. 4th ed. Thousand Oaks, CA: Sage; 2014:273 p.

14. Chaudakshetrin P. Validation of the Thai version of brief pain inventory (BPI-T) in cancer patients. J Med Assoc Thai. 2009;92(1):34-40.

15. Buppha P, Tontisirin N, Numthavaj P, Sakdanuwatwong S, Sodsee W, Finlayson RJ. Reliability and validity of the Thai short-form McGill pain questionnaire-2 (SF-MPQ-2). J Anesth Clin Res. 2016;7(8):651.

16. SPSS Inc. PASW Statistics for Windows, Version 18.0. Chicago: SPSS Inc; 2009.

17. Development SS. ATLAS.ti 8.0 Windows. Berlin: Scientific Software Development; 2017

18. Pope C, Ziebland S, Mays N. Qualitative research in health care. Analysing qualitative data. BMJ. 2000;320(7227):114-116.

19. LeMay K, Wilson KG, Buenger U, et al. Fear of pain in patients with advanced cancer or in patients with chronic noncancer pain. Clin J Pain. 2011;27(2):116-124.

20. Peres MF, Lucchetti G. Coping strategies in chronic pain. Curr Pain Headache Rep. 2010;14(5):331-338.

21. Dixon KE, Keefe FJ, Scipio CD, Perri LM, Abernethy AP. Psychological interventions for arthritis pain management in adults: a meta-analysis. Health Psychol. 2007;26(3):241-250.

22. Ballantyne J.C. Coping with pain. IASP Pain: Clinical update [online] 2009:11(5). Available from: https://www.iasp-pain.org/PublicationsNews/NewsletterIssue.aspx?ItemNumber=2094. Accessed April 1, 2017.

23. Rosenstiel AK, Keefe FJ. The use of coping strategies in chronic low back pain patients: relationship to patient characteristics and current adjustment. Pain. 1983;17(1):33-44.

24. Riley JL, Robinson ME. CSQ: five factors or fiction? Clin J Pain. 1997;13(2):156-162. 


\section{Supplementary material}

\section{Semistructured questions for focus-group interviews}

1. The experience in writing

- Have you ever had experience in writing before?

- If yes, please talk about your experience.

- What benefits did you get when writing?

2. Feeling after using pain diary

- What changes did you have after using the pain diary?

- How did you feel after using the pain diary?

- Did you get a benefit from using the pain diary? And why?

3. Recognizing pain

- How often did you recognize your pain?

- Why did you think that recognizing pain was important?

- How did your recognition change after using the pain diary?

4. Self-management of controlling pain

- How did you manage your pain?
- How did you use the pain diary for changing your pain management?

5. Sense of control

- Did you feel that you can control your pain?

- If yes, how did you get a sense of control?/If no, how did you not get sense of control?

- How did your sense of control change after using the pain diary?

6. Communication

- Who did you want to talk to about your pain/pain management? And why did you choose them?

- How did they react after talking about your pain?

- How did your relationship change after talking?

- How did you use the pain diary for communicating about pain?

- Did you feel more confident to communicate after using the pain diary?

7. Other changes that they had after using the pain diary

8. What were the problems when you used the pain diary?
Journal of Pain Research

\section{Publish your work in this journal}

The Journal of Pain Research is an international, peer reviewed, open access, online journal that welcomes laboratory and clinical findings in the fields of pain research and the prevention and management of pain. Original research, reviews, symposium reports, hypothesis formation and commentaries are all considered for publication.

\section{Dovepress}

The manuscript management system is completely online and includes a very quick and fair peer-review system, which is all easy to use. Visit http://www.dovepress.com/testimonials.php to read real quotes from published authors. 